\title{
УЧЕТ ФАКТОРОВ ВРЕМЕНИ ПРИ ОПТИМИЗАЦИИ ТОПЛИВНО- ЭНЕРГЕТИЧЕСКИХ БАЛАНСОВ ОБЪЕДИНЕНИЯ ЭКОНОМИЧЕСКИХ РАЙОНОВ НА ОСНОВЕ ПРИНЦИПОВ САМООРГАНИЗАЦИИ
}

G. RABKIN, K. JAANIMAGI. ISEORGANISEERIMISE PRINTSIIPIDE KASUTAMINE AJAFAKTORI ARVESTAMISEKS MAJANDUSRAJOONIDE KOONDISE KOTUSE-ENERGIABILANSSIDE OPTIMEERIMISEL

G. RABKIN, K. IAANIMAGI. THE USE OF THE SELF-ORGANIZING PRINCIPLES FOR TAKING INTO ACCOUNT THE TIME FACTOR IN OPTIMIZATION OF THE FUEL ENERGY BALANCE OF THE UNITED ECONOMICAL REGION

\section{(Представил И. Эпик)}

В $\left[{ }^{1,2}\right]$ учет фактора времени при оптимизации топливно-энергетических балансов (ТЭБ) экономических районов предлагается осуществлять на основе двухэтапного процесса. На первом этапе строится имитационная динамическая модель, на втором - с использованием полученных выше параметров формируется оптимизационная модель вида

$$
\begin{gathered}
\sum_{j=1}^{J} \sum_{i=1}^{n} \sum_{l=1}^{t} c_{i l j} x_{i l j} \rightarrow \min , \\
\sum_{j=1}^{J} \sum_{i=1}^{n} \sum_{l=1}^{t} a_{i l j}^{k} x_{i l j} \rightarrow \min , \\
\sum_{j=1}^{J} \sum_{i=1}^{n} \sum_{l=1}^{t} \mathrm{U}_{i l j} x_{i l j} \rightarrow \min , \\
\sum_{j=1}^{J} \sum_{i=1}^{n} \sum_{l=1}^{t} q_{i l j} x_{i l j} \rightarrow \min , \\
\sum_{i=1}^{n} \eta_{i l j} x_{i l j} \geqslant Q_{l j}, \\
\sum_{i=1}^{n} a_{i l j}^{k} x_{i l j} \leqslant Y_{l j}^{k}, \\
\sum_{l=1}^{t} x_{i l j}=x_{i j},
\end{gathered}
$$

где $j=\overline{1, J}$ - дискретный интервал планирования, остальные параметры $(1)-(7)$ описаны в $\left[{ }^{1}\right]$.

Используя решения задачи (1)-(7) для различных значений $x_{i j, r}\left(x_{i j, r}-\right.$ потребность $r$-го района в $i$-м топливе в $j$-й год), можно построить обобщенные характеристики $\left[{ }^{1}\right]$ ТЭБ $r$-го района. 


$$
z_{r}=\Psi\left(x_{i j, r}\right) \text {. }
$$

Очевидно, что обобщенная характеристика ТЭБ (8) имеет значительное число переменных, и поэтому применение традиционных методов регрессионного анализа для построения этой характеристики не представляется возможным, так как регрессионный аналлиз позволяет строить модели только в области, где число коэффициентов модели равно или меньше числа точек таблицы опытных данных. В силу этого обстоятельства построение обобщенной характеристики ТЭБ предлагается осуществлять на основе метода группового учета аргументов (МГУА), использующего многорядные алгоритмы самоорганизации $\left[{ }^{3,4}\right]$. Эти алгоритмы позволяют найти структуру модели оптимальной сложности соответствующей минимуму некоторого внешнего критерия, т. е. критерия, определение которого основано на информации, неиспользованной при синтезе модели.

Пусть структура обобщенной характеристики ТЭБ (8) определена и является полиномом второй степени. После перехода в модели (8) к одноиндексным переменным и линеаризации переменных старших степеней получим линейную модель обобщенной характеристики следующего вида

$$
z=\alpha_{0}+\sum_{m=1}^{S} \alpha_{m} y_{m}
$$

где

$$
\begin{aligned}
& y_{m}=x_{i j}, \quad i=\overline{1, n}, \quad j=\overline{1, J}, \quad m=j+(i-1) J, \quad m=\overline{1, n J}, \\
& y_{m+p}=y_{p}^{2}, \quad p=\overline{1, n J}, \\
& y_{2 m+q}=y_{p_{1}} \cdot y_{p_{2}}, \quad p_{1}<p_{2}, \quad p_{2}=\overline{2, n J} .
\end{aligned}
$$

Очевидно, что число переменных в модели $\quad S=\frac{n J(n J+3)}{2}$.

Далее, при использовании многорядного алгоритма МГУА для определения коэффициентов модели (9) на первом ряду селекции образуются все возможные комбинации по два аргумента и для каждой из этих пар находится частное описание в виде линейной модели

$$
Y_{h}=Y_{h}\left(y_{m_{1}}, y_{m_{2}}\right)=a_{0_{h}}+a_{1_{h}} y_{m_{1}}+a_{2_{h}} y_{m_{2}} .
$$

Здесь $\quad h=\overline{1,} C_{s}^{\overline{2}}, \quad m_{1}<m_{2}, \quad m_{1}=\overline{1, S-1}, \quad m_{2}=\overline{2, S}$.

Из всех частных моделей вида (10) выбираются $F=S$ лучших, дающих минимальное значение внешнего критерия (критерия селекции). Во втором ряду селекции образуются комбинации по два выходных переменных прошедших первый ряд, и для каждой из них определяется частное описание типа (10) второго ряда и т. д. Наращивание рядов селекции происходит до тех пор пока критерий селекции не достигнет своего минимума. На последнем ряду селекции выбирается модель, соответствующая этому минимуму.

Очевидно, что при линейных частных моделях степень полинома не увеличивается на каждом ряду селекции, меняется только число членов входящих в окончательный полином. Поэтому этот полином в силу (9) будет являться полиномом второго порядка, что и требуется.

В качестве критерия селекции в данном случае нами рекомендуется к р и тери й регуля рности. Этот критерий основан на разделенй имеющихся $u=\overline{1, N}$ экспериментальных данных на две части: обучаю- 
щую $N_{A}$ и проверочную $N_{B}$ последовательность точек. Оценки коэффициентов рассчитываются по обучающей последовательности. Критерий регулярности представляет собой среднеквадратичную ошибку на проверочной последовательности точек, не использованных для получения оценок коэффициентов частных описаний:

$$
\Delta^{2}(B)=\frac{\sum_{u=1}^{N_{B}}\left(f_{\text {табл }}-f_{\mathrm{M}}\right)_{u}^{2}}{\sum_{u=1}^{N_{B}}\left(f_{\text {табл }}^{2}\right)_{u}} \rightarrow \text { min, }
$$

где $f_{\text {табл }}-$ табличное значение выходной переменной, $f_{\text {м }}-$ значения, рассчитанные по данной модели.

Использование критерия регулярности в качестве внешнего критерия основано на том обстоятельстве, что он ориентирован на модель, которая будет наиболее точной на множестве точек, которых еще нет в таблице данных, что собственно и является основной задачей прогнозирования.

Для получения оптимальных количеств эффективных видов топлива, выделяемых $r$-му району в $j$-й год необходимо решить многоцелевую задачу квадратичного программирования

$$
\begin{aligned}
& z_{r} \rightarrow \min \quad r=\overline{1, R} \\
& \sum_{r=1}^{R} x_{i j, r}=x_{i j} .
\end{aligned}
$$

Оптимальные количества эффективных видов топлива, выделяемые потребителем в $j$-й год планирования, получаются на основе подстановки оптимальных компромиссных решений вышеприведенной целевой задачи квадратичного программирования в четырехцелевую задачу оптимизации ТЭБ района и ее последующего решения.

\section{ЛИТ Е РАТ У РА}

1. Вайк Л. Э., Рабкин $Г$. Б., Яанимяги К. Э. Согласованная оптимизация топливноэнергетических балансов экономических районов: теория и методы. Таллин, Изд. АН ЭССР, 1982.

2. Яанимяги К.-Изв. АН ЭССР. Физ. Матем., 30, № 3, 281-283 (1981).

3. Нвахненко А. Г., Зайченко Ю. П., Димитров В. Д. Принятие решений на основе самоорганизации. М., «Советское радио», 1976.

4. Ивахненко A. $\Gamma$. Индуктивный метод самоорганизации моделей сложных систем. Киев, «Наукова думка», 1982.

Ннститут термофизики и электрофизики Академии наук Эстонской ССР
Поступила в редакцию 29/III 1983 